\title{
Association of time to craniectomy with survival in patients with severe combat-related brain injury
}

\author{
Stacy A. Shackelford, MD, ${ }^{1}$ Deborah J. del Junco, PhD, ${ }^{1,2}$ Michael C. Reade, MBBS, DPhil, ${ }^{3}$ \\ Randy Bell, MD, ${ }^{4}$ Tyson Becker, MD, ${ }^{5}$ Jennifer Gurney, MD, ${ }^{1}$ Randall McCafferty, MD, ${ }^{6}$ and \\ Donald W. Marion, MD'
}

${ }^{1}$ Joint Trauma System, Defense Center of Excellence, San Antonio; ${ }^{2 D e p a r t m e n t ~ o f ~ E p i d e m i o l o g y ~ a n d ~ B i o s t a t i s t i c s, ~ U n i v e r s i t y ~ o f ~}$ Texas Health Science Center, San Antonio, Texas; ${ }^{3}$ Joint Health Command, Australian Defence Force, Brisbane, Queensland, Australia; ${ }^{4}$ Department of Neurosurgery, Walter Reed National Military Medical Center, Bethesda, Maryland; Departments of ${ }^{5}$ Surgery and ${ }^{6}$ Neurosurgery, San Antonio Military Medical Center, San Antonio, Texas; and ${ }^{7}$ Defense and Veterans Brain Injury Center, Silver Spring, Maryland

OBJECTIVE In combat and austere environments, evacuation to a location with neurosurgery capability is challenging. A planning target in terms of time to neurosurgery is paramount to inform prepositioning of neurosurgical and transport resources to support a population at risk. This study sought to examine the association of wait time to craniectomy with mortality in patients with severe combat-related brain injury who received decompressive craniectomy.

METHODS Patients with combat-related brain injury sustained between 2005 and 2015 who underwent craniectomy at deployed surgical facilities were identified from the Department of Defense Trauma Registry and Joint Trauma System Role 2 Registry. Eligible patients survived transport to a hospital capable of diagnosing the need for craniectomy and performing surgery. Statistical analyses included unadjusted comparisons of postoperative mortality by elapsed time from injury to start of craniectomy, and Cox proportional hazards modeling adjusting for potential confounders. Time from injury to craniectomy was divided into quintiles, and explored in Cox models as a binary variable comparing early versus delayed craniectomy with cutoffs determined by the maximum value of each quintile (quintile 1 vs $2-5$, quintiles $1-2$ vs $3-5$, etc.). Covariates included location of the facility at which the craniectomy was performed (limited-resource role 2 facility vs neurosurgically capable role 3 facility), use of head CT scan, US military status, age, head Abbreviated Injury Scale score, Injury Severity Score, and injury year. To reduce immortal time bias, time from injury to hospital arrival was included as a covariate, entry into the survival analysis cohort was defined as hospital arrival time, and early versus delayed craniectomy was modeled as a time-dependent covariate. Follow-up for survival ended at death, hospital discharge, or hospital day 16 , whichever occurred first.

RESULTS Of 486 patients identified as having undergone craniectomy, $213(44 \%)$ had complete date/time values. Unadjusted postoperative mortality was $23 \%$ for quintile $1(n=43$, time from injury to start of craniectomy 30-152 minutes); $7 \%$ for quintile 2 ( $n=42,154-210$ minutes); $7 \%$ for quintile $3(n=43,212-320$ minutes); $19 \%$ for quintile 4 ( $n=42$, 325-639 minutes); and $14 \%$ for quintile $5(n=43,665-3885$ minutes). In Cox models adjusted for potential confounders and immortal time bias, postoperative mortality was significantly lower when time to craniectomy was within 5.33 hours of injury (quintiles $1-3$ ) relative to longer delays (quintiles $4-5$ ), with an adjusted hazard ratio of $0.28,95 \% \mathrm{Cl} 0.10-0.76$ $(p=0.012)$.

CONCLUSIONS Postoperative mortality was significantly lower when craniectomy was initiated within 5.33 hours of injury. Further research to optimize craniectomy timing and mitigate delays is needed. Functional outcomes should also be evaluated.

https://thejns.org/doi/abs/10.3171/2018.9.FOCUS18404

KEYWORDS craniectomy; military trauma system; timing of craniectomy; severe traumatic brain injury

ABBREVIATIONS AIS = Abbreviated Injury Scale; DECRA = Decompressive Craniectomy trial; ED = Emergency Department; GCS = Glasgow Coma Scale; ICP = intracranial pressure; IQR = interquartile range; ISS = Injury Severity Score; RESCUE-ASDH = Randomized Evaluation of Surgery with Craniectomy for patients Undergoing Evacuation of Acute Subdural Hematoma; RESCUEicp = Randomized Evaluation of Surgery with Craniectomy for Uncontrollable Elevation of intracranial pressure; $\mathrm{TBI}=$ traumatic brain injury.

ACCOMPANYING EDITORIAL DOI: 10.3171/2018.9.FOCUS18497.

SUBMITTED August 9, 2018. ACCEPTED September 12, 2018.

INCLUDE WHEN CITING DOI: 10.3171/2018.9.FOCUS18404. 
$\mathrm{T}$ RAUMATIC brain injury (TBI) is a leading cause of death and disability among military and civilian patients injured in combat. ${ }^{24}$ One-third of battlefield deaths have been attributed to TBI, ${ }^{10}$ and among those considered preventable that have occurred after arrival at a surgical hospital, $9 \%$ have been attributed to brain injury. ${ }^{9}$

Survival after severe TBI depends on prehospital stabilization; optimization of medical treatments to control elevated intracerebral pressure; ${ }^{27}$ and, for those with expanding intracranial mass lesions, emergency craniectomy for evacuation of the hematoma or decompression of the swollen brain. Thus, severely brain-injured patients may benefit from early neurosurgical intervention. Although it has been shown that early physiological stabilization to avoid hypoxia, hypotension, and hypo- and hypercarbia led to improved outcomes from TBI, ${ }^{34-36}$ the optimum time window for neurosurgical intervention in patients with TBI remains uncertain.

In 1981, Seelig et al. ${ }^{32}$ reported that among 82 patients with acute subdural hematoma who underwent decompressive craniectomy, severe morbidity and mortality was 3 times greater $(\mathrm{p}<0.0001)$ in those whose surgery was delayed more than 4 hours after injury. Based on this finding, the American College of Surgeons Committee on Trauma recommended that patients undergoing a craniotomy more than 4 hours after Emergency Department (ED) admission be audited for opportunities to improve care. ${ }^{5}$ In 1999, a Royal College of Surgeons report included the recommendation that "life-saving decompressive surgery must be available for all patients who require it within four hours of injury," although no original source reference was cited. ${ }^{31}$ In reports of at least 25 other observational studies published since 1968 that addressed the relationship between time to emergency neurosurgery (in hours) and outcomes, 19 of the studies concluded that shorter delays were not associated with better survival or neurological outcome, $3,6,7,14,15,17,19,21-23,25,28,29,33,37-39,41-44$ whereas only 6 supported the conclusion that delays of less than 3-5 hours were associated with improved outcomes. ${ }^{12,13,26,30,40,45} \mathrm{Re}$ cent systematic reviews ${ }^{1,18}$ concluded that the time point after which outcomes deteriorate remains uncertain, and called for more definitive studies to resolve the conflicting findings in the literature.

The association between time to neurosurgery and survival has not been evaluated in the population of patients with combat-related TBI. ${ }^{8}$ From a military trauma system and planning perspective, a target in terms of the optimum timeline within which to initiate neurosurgery is of paramount importance to inform the strategic allocation of neurosurgical assets and transport resources to support a population at risk. This study sought to identify a population of patients with severe combat-related brain injuries who received decompressive craniectomy, and to examine the association of time to craniectomy with mortality.

\section{Methods}

This performance improvement project was deemed exempt from institutional review board approval by the Department of Defense Joint Trauma System. US military and non-US military patients injured in combat during the years 2005-2015 who underwent decompressive craniectomy at a deployed surgical facility (either role 2 or role 3) in Iraq or Afghanistan were identified from the Department of Defense Trauma Registry and Joint Trauma System Role 2 Registry. In a military trauma system, role 2 facilities provide forward resuscitative care with limited resources, whereas role 3 hospitals provide definitive theater support, usually including neurosurgery capabilities. Patients eligible for the study survived transport to a hospital capable of diagnosing the need for craniectomy and performing the surgery. From the electronic data resources available, it was not possible to ascertain if any patients who were diagnosed with an indication for craniectomy died before the start of surgery.

Statistical analyses included univariate comparisons of patients who underwent craniectomy with and without complete date and time data by using chi-square, Wilcoxon rank-sum tests, and multivariable logistic regression. Among patients with nonmissing date and time data, postoperative mortality was compared in unadjusted analyses. These analyses involved chi-square tests for overall differences across quintiles of the elapsed time from injury occurrence to the start of craniectomy (in hours), and Cox proportional hazards regression to adjust survival analyses for potential confounders. The correlation between elapsed time from injury to craniectomy (in quintiles) and severity of head injury (maximum Abbreviated Injury Scale [AIS] score) was assessed using Spearman's rank correlation. The quintiles of elapsed time from injury to craniectomy were combined in the Cox models to form binary variables and compare early versus delayed craniectomy, with cutoffs determined by the maximum value of each successive quintile (i.e., quintile 1 vs 2-5, quintiles $1-2$ vs 3-5, etc.).

The following covariates were also included: location of craniectomy (role 2 vs role 3 facility), use of head CT scan, US military status, age, maximum head AIS score, Injury Severity Score (ISS), and year of injury. To reduce immortal time bias as much as possible, time from injury occurrence to hospital arrival (in hours) was included as a covariate, entry into the survival analysis cohort (time 0 ) was defined as hospital arrival time, and early versus delayed craniectomy, as defined above, was modeled as a time-dependent covariate $(1=$ early, $0=$ still waiting/ delayed). Follow-up for survival ended at death, hospital discharge, or hospital day 16, whichever occurred first. Follow-up extended to at least 1 day after hospital arrival for $95 \%$ of survivors, 2 days for $85 \%, 5$ days for $50 \%$, and 16 days for $15 \%$. Survivors discharged before day 16 were considered alive. The duration of follow-up was not significantly associated with the time to craniectomy $(\mathrm{p}=0.54)$, which supports the validity of the survival analyses.

Delays to surgery (in hours) among patients transferred from a role 2 to a role 3 facility where the craniectomy was performed were compared with delays among the patients with craniectomy who were not transferred. Nonparametric median score tests were applied to assess the betweengroup differences for overall delays (from injury to craniectomy) as well as the prehospital and in-hospital segments.

Mortality after craniectomy performed at a role 2 or 3 facility without a neurosurgeon (with or without CT 
scanner) was compared to mortality after craniectomy performed at a role 3 facility with both CT scanner and neurosurgeon available in the full cohort of patients who underwent craniectomy, regardless of missing date/time values, by using multiple logistic regression to adjust for important covariates including age, US military status, year of injury, time elapsed from injury occurrence to arrival at the hospital in which craniectomy was performed, head CT scan, maximum head AIS score, and ISS. Other covariates considered (sex, mechanism of injury, initial Glasgow Coma Scale [GCS] score, initial systolic blood pressure, initial heart rate, subdural or epidural hematoma, and penetrating brain injury) were not independent mortality risk factors in either the logistic or Cox survival analysis models, and changed the estimated associations with mortality less than $15 \%$. Interaction terms included in the logistic and Cox models to test for potential effect modification by individual covariates (mechanism of injury, penetrating brain injury) were not significant. Probability values less than 0.05 were considered statistically significant.

\section{Results}

A total of 486 patients who had undergone craniectomy were identified, and date/time values for injury occurrence, hospital arrival, start of craniectomy, and death or hospital discharge were available for 213 (44\%). Of the 273 patients with missing time data, 257 had no time of arrival at the hospital in which craniectomy was performed, 236 had no time of injury, 40 had no neurosurgery start time, and 2 had no time of postoperative death; 236 were missing more than one time point. Injury times were more often missing for the non-US military patients (189/375 [50\%] vs 47/111 [42\%]). The proportion of missing injury times decreased over the study time period, from $49 \%$ in 2005-2007 to 27\% in 2012-2015.

Demographic, injury, and clinical characteristics for the total group of 486 patients and the subgroup of 213 study patients who had complete date and time data were compared (Table 1). The two independent subgroups (with and without complete date/time data) did not differ significantly in terms of outcomes, discharge GCS score ( $p$ $=0.55)$, or death $(\mathrm{p}=0.40)$, even after adjusting for the set of covariates listed in Table 1 ( $p=0.48, p=0.66$, respectively). These findings support the validity of the restricted complete-case survival analysis using time-dependent Cox proportional hazards modeling.

For the 213 patients with complete date/time values, prehospital delays from injury to arrival at the hospital in which craniectomy was performed were $71 \%$ longer, on average, than their in-hospital delays from hospital arrival to the start of neurosurgery (Table 1). Table 2 provides the range of total elapsed times, from injury to the start of craniectomy, for the 213 time-study patients subdivided into quintiles. In unadjusted analyses, neither the overall association among the quintiles nor the linear trend with increasing quintiles was significant $(p=0.12, p=$ 0.68 ). Severity of head injury, measured by the maximum head AIS score, was inversely correlated with increasing quintiles of the total time to craniectomy (Spearman's $\rho$
$=-0.14, \mathrm{p}=0.038)$. However, maximum head AIS score was significantly correlated only with patients' in-hospital delays to surgery (Spearman's $\rho=-0.19, p=0.006$ ), not their prehospital delays (Spearman's $\rho=-0.03, p=0.66$ ). These findings highlight the importance of adjusting the time-dependent Cox models for both patients' injury severity and prehospital delays to surgery to allow for the important individual and joint effects of these factors.

In the time-dependent Cox models adjusted for potential confounders and immortal time bias, postoperative mortality was significantly lower when the time to craniectomy was within 5.33 hours of injury (quintiles 1-3) relative to longer delays (quintiles $4-5$ ); adjusted hazard ratio $0.28,95 \%$ CI $0.10-0.76(\mathrm{p}=0.012)$ (Fig. 1). Betweengroup mortality differences for all of the other quintile contrasts (quintile 1 vs $2-5,1-2$ vs $3-5,1-4$ vs 5 ) were not statistically significant. Ignoring the prehospital delays and focusing strictly on study patients' delays to neurosurgery after hospital arrival demonstrated no significant association between earlier craniectomy and reduced mortality; adjusted hazard ratio $0.80,95 \%$ CI $0.30-2.16$ ( $\mathrm{p}=$ 0.66) (Fig. 2).

The 63 patients who underwent craniectomy at a role 3 hospital following transfer from a role 2 facility had significantly longer delays to neurosurgery than the 150 patients who had not been transferred (including the 9 patients who underwent craniectomy at the role 2 facility). The median delays from injury to craniectomy and the interquartile ranges (IQRs) for patients transferred from role 2 to role 3 hospitals versus those who were not transferred were 7.1 (IQR 4.3-13.1) versus 3.5 (IQR 2.5-6.5) hours, respectively $(p<0.0001)$. The median prehospital delays (from injury to arrival at the hospital in which craniectomy was performed) comparing transferred with nontransferred patients were 4.2 (IQR 2.5-7.3) versus 2.0 (IQR 0.9-3.9) hours ( $<0.0001)$. The median in-hospital delays to neurosurgery comparing transferred with nontransferred patients were 1.8 (IQR 1.0-3.6) versus 1.3 (IQR 0.9-2.1) hours $(\mathrm{p}=0.022)$.

Among the total of 486 patients, 36 underwent craniectomy at a facility with no neurosurgeon available (26 also had no access to a CT scanner), whereas 450 craniectomies were performed at a role 3 facility with both CT scanner and neurosurgeon available (Table 1). The unadjusted postoperative mortality rate was $25 \%$ for craniectomy without neurosurgeon versus $15 \%$ for craniectomy with neurosurgeon $(\mathrm{p}=0.148)$. After adjustment for the key covariates, the mortality odds ratio following craniectomy performed by non-neurosurgeons compared to neurosurgeons dropped below the null to 0.89 (95\% CI $0.29-2.69, \mathrm{p}=0.830$ ).

\section{Discussion}

For seriously brain-injured patients, survival with best neurological outcome depends on optimal treatment from the point of injury through evacuation (including any interfacility transfers), to arrival at a neurosurgically capable hospital; accurate diagnosis of the indications for surgery; and initiation of the appropriate procedures. For patients with severe combat-related TBI who underwent craniec- 
TABLE 1. Demographic and clinical characteristics of 486 patients undergoing craniectomy

\begin{tabular}{|c|c|c|}
\hline Demographic or Clinical Characteristic & Total Study Population, N = 486 & Subgroup for Timing Analysis, $n=213^{*}$ \\
\hline Median age in yrs (IQR) & $25(21,31)$ & $25(21,32)$ \\
\hline Sex (\% male) & $471(97 \%)$ & $211(99 \%)$ \\
\hline US military & $111(23 \%)$ & $49(23 \%)$ \\
\hline Median year of injury (IQR) & $2008(2006,2010)$ & $2008(2006,2011)$ \\
\hline \multicolumn{3}{|l|}{ Mechanism of injury } \\
\hline GSW & $168(35 \%)$ & $83(39 \%)$ \\
\hline Explosives & $239(49 \%)$ & $95(45 \%)$ \\
\hline Other & $79(16 \%)$ & $35(16 \%)$ \\
\hline Theater of operation & $n=472$ & $n=212$ \\
\hline Afghanistan & $200(42 \%)$ & $101(48 \%)$ \\
\hline Iraq & $272(58 \%)$ & $111(52 \%)$ \\
\hline \multicolumn{3}{|l|}{ Craniectomy location } \\
\hline Role 2 vs role 3 facility & $36(7 \%)$ & $9(4 \%)$ \\
\hline Role 3 patients transferred from role 2 facility & $122(27 \%) ; n=450$ & $63(31 \%) ; n=204$ \\
\hline Head CT scan & $423(87 \%)$ & $204(96 \%)$ \\
\hline Penetrating brain injury & $209(43 \%)$ & $101(47 \%)$ \\
\hline Hematoma, epidural or subdural & $349(72 \%)$ & $162(76 \%)$ \\
\hline Median maximum head AIS score (IQR) & $4(3,5)$ & $4(4,5)$ \\
\hline Median ISS (IQR) & $25(17,30)$ & $25(17,30)$ \\
\hline Median initial total GCS score (IQR) & $3(3,10) ; n=396$ & $3(3,10) ; n=178$ \\
\hline Median initial heart rate (IQR) & $89(75,106) ; n=388$ & $84(72,100) ; n=176$ \\
\hline Median initial SBP (IQR) & $128(111,141) ; n=387$ & $125(110,140) ; n=176$ \\
\hline Median hrs (IQR) & $n=213$ & $n=213$ \\
\hline From injury occurrence to arrival at craniectomy facility & $2.5(1.0,5.0)$ & $2.5(1.0,5.0)$ \\
\hline From arrival at craniectomy facility to start of craniectomy & $1.5(0.9,2.3)$ & $1.5(0.9,2.3)$ \\
\hline From injury occurrence to craniectomy & $4.4(2.8,8.5)$ & $4.4(2.8,8.5)$ \\
\hline In-hospital death & $76(16 \%)$ & $30(14 \%)$ \\
\hline Discharge GCS score (total) & $n=298$ & $n=136$ \\
\hline Median (IQR) & $9(3,15)$ & $9(3,15)$ \\
\hline
\end{tabular}

GSW = gunshot wound; SBP = systolic blood pressure.

Unless otherwise indicated, values are expressed as the number of patients (\%).

* Subgroup was $44 \%$ of 486 .

tomy, surgery performed within 5.33 hours of injury was associated with significantly reduced mortality compared to longer delays. Adjusted survival analyses balanced the

TABLE 2. In-hospital mortality by quintiles of time to craniectomy from injury occurrence

\begin{tabular}{cccc}
\hline $\begin{array}{c}\text { Group } \\
\text { (quintile) }\end{array}$ & $\begin{array}{c}\text { Total } \\
(\mathrm{N}=213)\end{array}$ & $\begin{array}{c}\text { Range of Elapsed } \\
\text { Time to Craniectomy }\end{array}$ & $\begin{array}{c}\text { No. (\%) w/ In-Hospital } \\
\text { Mortality; Unadjusted }\end{array}$ \\
\hline 1 & 43 & $0.5-2.5 \mathrm{hrs}$ & $10(23 \%)$ \\
\hline 2 & 42 & $2.6-3.5 \mathrm{hrs}$ & $3(7 \%)$ \\
\hline 3 & 43 & $3.5-5.3 \mathrm{hrs}$ & $3(7 \%)$ \\
\hline 4 & 42 & $5.4-10.7 \mathrm{hrs}$ & $8(19 \%)$ \\
\hline 5 & 43 & $11.0 \mathrm{hrs}-2.7$ days & $6(14 \%)$ \\
\hline
\end{tabular}

$\chi^{2}=7.31$ for overall differences, $p=0.12$. Nonparametric test for linear trend, $z$ $=-0.42, p=0.68$. between-group differences in injury severity and other covariates, accounted for the time dependency of emergency neurosurgical intervention, and revealed how longer prehospital delays were more significantly associated with postoperative mortality than in-hospital delays.

Transfer from a role 2 to a role 3 hospital where craniectomy was ultimately performed added significant prehospital and in-hospital delays relative to the other neurosurgeries without prior transfer. In adjusted analyses that included the entire cohort of 486 patients, the short-term outcome of postoperative mortality was roughly equal after emergency craniectomy, regardless of availability of a neurosurgeon or CT scanner, although only a limited number of craniectomies $(n=36)$ were performed by non-neurosurgeons. Taken together, the findings from this study suggest that the time from injury to craniectomy is of paramount importance for postoperative survival, perhaps even if the procedure is performed by a nonexpert.

Ideally, patients with severe combat-related TBI will 


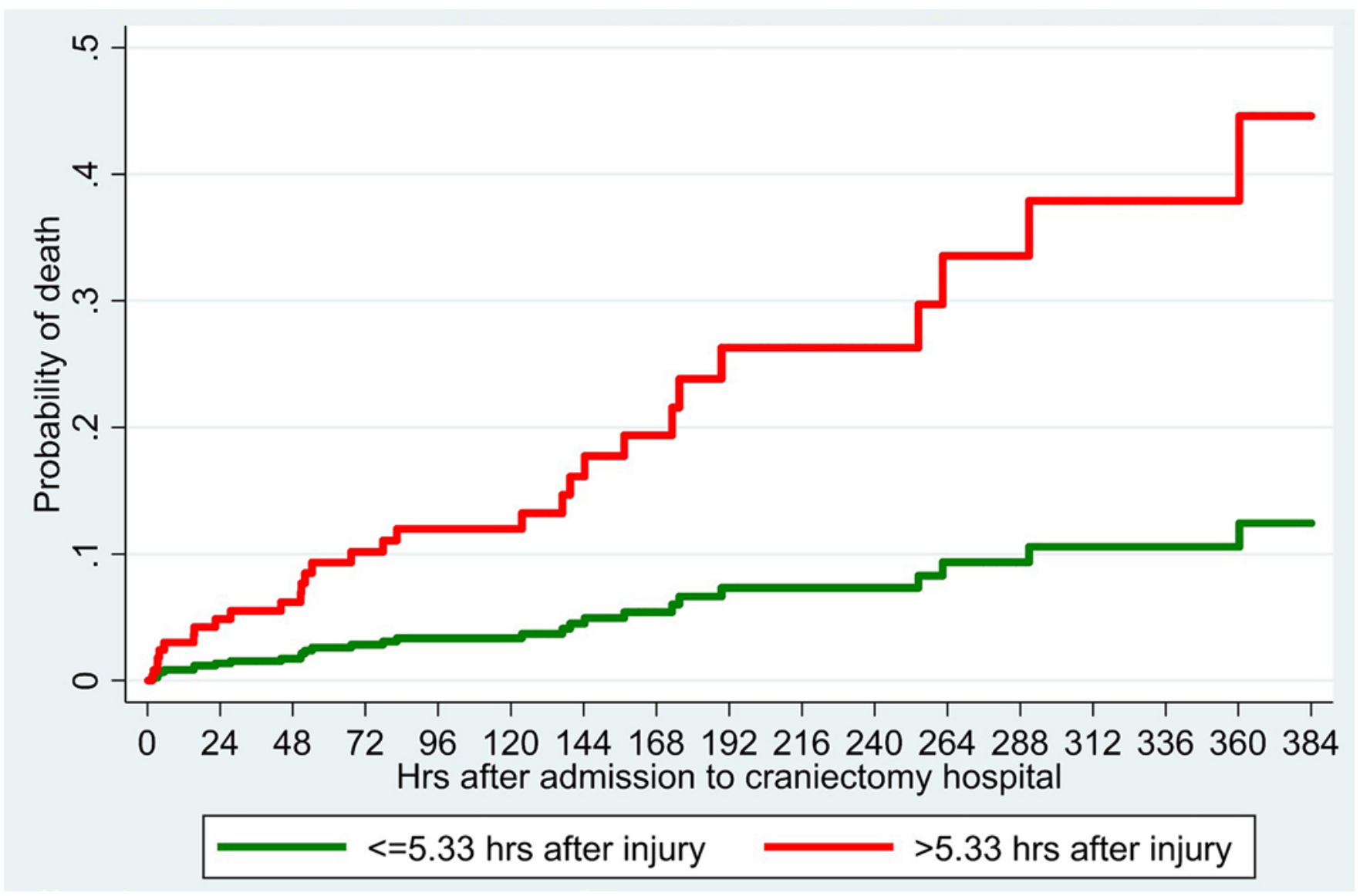

FIG. 1. Cox proportional hazards regression, time from injury to craniectomy. Adjusted mortality curves by Cox proportional hazards regression at the median value of each of the following covariates (see Table 1): location of craniectomy (limited-resource role 2 facility vs neurosurgically capable role 3 facility), use of head CT scan, US military status, age, maximum head AIS score, ISS, year of injury, and time from injury occurrence to hospital arrival (in hours). Entry into the survival analysis cohort (time 0 ) was defined as hospital arrival time, and patients' start of craniectomy was modeled as a time-dependent covariate and classified as follows: early, $\leq 5.33$ hours after injury (quintiles 1-3, Table 2); or delayed, $>5.33$ hours after injury (quintiles 4-5, Table 2). Followup for survival ended at death, hospital discharge, or hospital day 16, whichever occurred first. Other baseline characteristics listed in Table 1 were evaluated as candidate covariates, but were either not independent predictors of mortality or changed the hazard ratio for early versus delayed craniectomy less than $10 \%$.

reach a neurosurgically capable hospital within hours of injury. However, the realities of worldwide military operations and potentially vast distances of travel may prohibit timely transport to a definitive level of care. In such cases, providers must weigh the risks versus benefits of transport to a distant, neurosurgically capable military hospital; a less remote host-nation facility; or a nearby, resource-limited facility in an austere environment capable of providing neurosurgical intervention only by a general surgeon with uncertain access to expert teleconsultation. ${ }^{2}$ Military providers inevitably face these exigencies, and valid, precise estimates of the upper limit of the optimal time to emergency neurosurgery for patients with severe combat-related TBI can help to improve decision-making. Our study does not define 5.33 hours as a goal (because there are most likely benefits to swifter intervention), but it does suggest that for patients who require craniectomy, 5.33 hours from injury to craniectomy is a timeline ideally not to be exceeded, beyond which mortality increases.

Although faster time to surgery for the 2 lowest quintil- es (with cutoffs of either 2.5 or 3.5 hours) was not shown to be significant, 8,10 this observational study was underpowered to detect more subtle effects and excluded any patients with potential indication for craniectomy who died before surgery was possible (which would have biased the hazard ratios for the lowest quintiles upward, countering any true protective effect, given that most trauma deaths occur in the minutes to early hours after injury). If there were any neurosurgery-eligible patients whose early death precluded craniectomy, their inclusion in our study could only have strengthened the observed significantly protective association for shorter delays (within 5.33 hours). Without diagnostic data (e.g., intracranial pressure [ICP] monitoring), appropriate delays for the more slowly evolving surgical indications could not be distinguished from potentially life-threatening delays for the patients with more immediate indications, adding noise from misclassification that tends to weaken associations. Including the covariate for prehospital delays along with the separate analysis focused solely on in-hospital delays helped ad- 


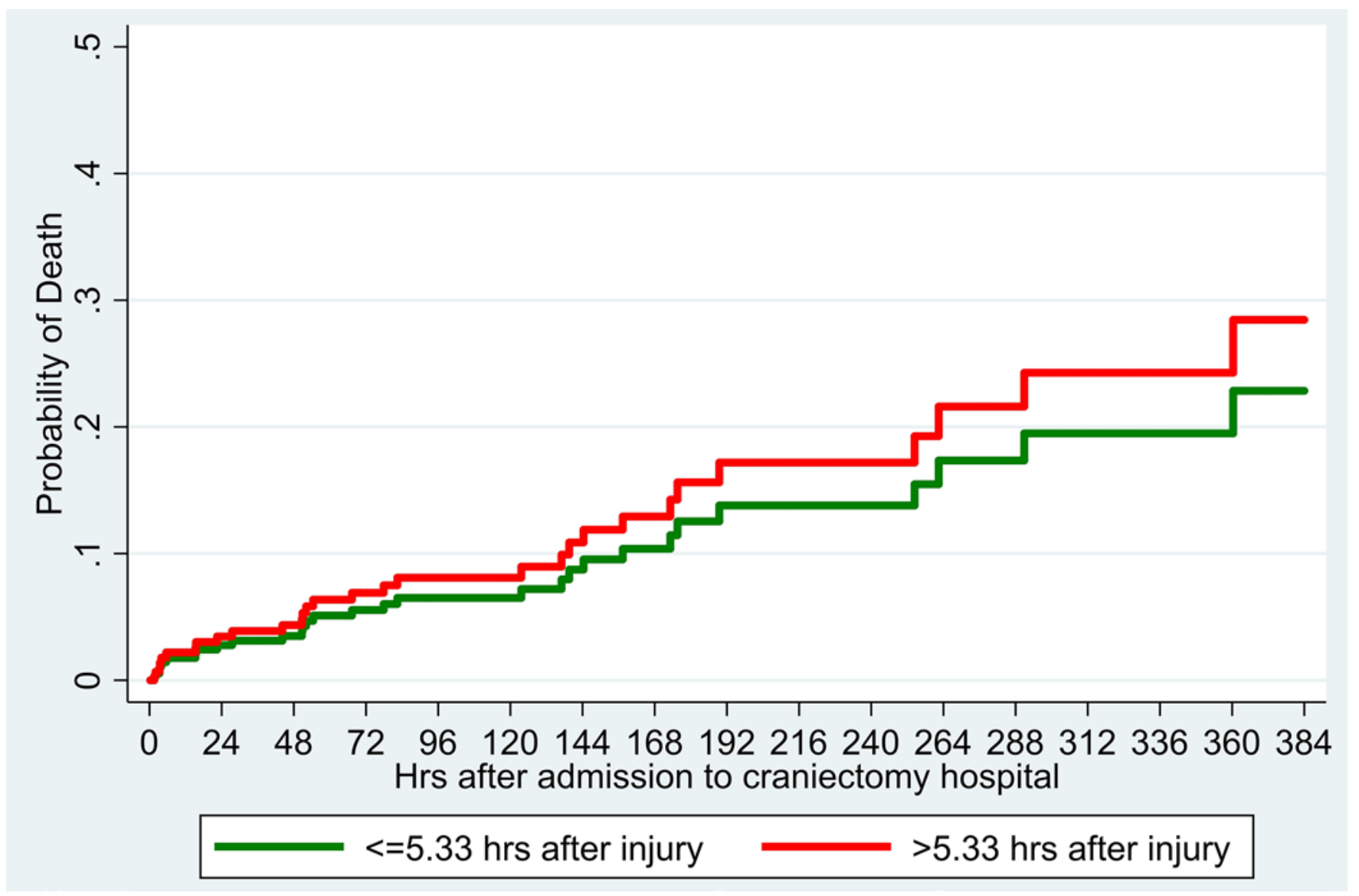

FIG. 2. Cox proportional hazards regression, time from ED arrival to craniectomy. Adjusted mortality curves by Cox proportional hazards regression excluding the covariate time from injury to hospital arrival, and setting each of the following covariates at their median value (Table 1): location of craniectomy (limited-resource role 2 facility vs neurosurgically capable role 3 facility), use of head CT scan, US military status, age, maximum head AIS score, ISS, and year of injury. Entry into the survival analysis cohort (time 0 ) was defined as hospital arrival time, and patients' start of craniectomy was modeled as a time-dependent covariate and classified as follows: early, $\leq 5.33$ hours after injury (quintiles $1-3$, Table 2); or delayed, $>5.33$ hours after injury (quintiles 4-5, Table 2). Follow-up for survival ended at death, hospital discharge, or hospital day 16, whichever occurred first. Other baseline characteristics listed in Table 1 were evaluated as candidate covariates, but were either not independent predictors of mortality or changed the hazard ratio for early versus delayed craniectomy less than $10 \%$.

dress this issue. In any case, our data support improving prehospital resources to expedite transport to neurosurgical care as quickly as possible and reduce the risk of mortality in combat TBI.

Previous observational studies of the effects of time to craniotomy or craniectomy have had inconsistent results with significant heterogeneity in study design. ${ }^{18}$ In particular, the delay to neurosurgery in previous studies has included various combinations of prehospital time, interfacility transfer time, and in-hospital time after reaching a place with neurosurgical capability. The time point used to calculate delays to neurosurgery has been variously defined as the injury occurrence time, $3,7,11,17,19,21-23,25,29,30,32,33$, 37-45 time of the call received by the emergency response system, ${ }^{6}$ time of patients' ED arrival, ${ }^{3,15,26,37,38}$ onset of the loss of consciousness, ${ }^{13,28}$ and onset of traumatic decerebration. ${ }^{12}$

Although all patients in the present study were treated with decompressive craniectomy, it remains controversial whether this procedure, when done specifically for elevated ICP, improves outcomes. The Decompressive Craniectomy (DECRA) randomized trial for severe TBI with refractory intracranial hypertension paradoxically demonstrated that improved ICP after craniectomy was not associated with improved survival or neurological outcome. ${ }^{4}$ However, all patients enrolled in DECRA had to have at least 12 hours of prerandomization (precraniectomy) ICP monitoring, and the median time from injury to surgery was 35.2 hours (IQR 23.3-52.8). Subsequently, the Randomized Evaluation of Surgery with Craniectomy for Uncontrollable Elevation of intracranial pressure (RESCUEicp) trial raised further questions about the benefit of decompressive craniectomy after finding that improved survival was countered by worse long-term neurological outcomes in patients with severe TBI treated with decompression. ${ }^{16}$ However, patients enrolled in the RESCUEicp trial had to have 1-6 hours of unremitting elevated ICP prior to randomization. With a median time from randomization to craniectomy of 2.2 hours, few if any patients in the RESCUEicp trial could have possibly 
received craniectomy within 5.3 hours of injury. In addition, the RESCUEicp trial evaluated only secondary, not primary decompressive craniectomy, which is the focus of the ongoing Randomized Evaluation of Surgery with Craniectomy for patients Undergoing Evacuation of Acute Subdural Hematoma (RESCUE-ASDH) trial. ${ }^{20}$ Neither of the completed trials evaluated outcomes in relation to the time from injury to surgery.

The systematic review by Barthélemy et al. ${ }^{1}$ concluded from retrospective studies that surgical intervention within 5 hours was associated with improved outcomes, particularly when other favorable indicators, such as age $<40$ years and GCS score 6-8 (vs 3-5), were present. These studies also highlight the fact that mortality alone is a poor measure of success in severe TBI, and further study including functional outcomes in patients with combatrelated TBI is needed. Strengths of the current observational study include a reasonably large sample size and accurate timelines to tease apart the separate influences of prehospital versus in-hospital delays while adjusting for immortal time bias, injury severity, and other important confounders. Prospective studies can better avoid immortal time bias by enrolling patients after treatment eligibility has been established, but before the study intervention is initiated (e.g., at randomization) and using intent-to-treat analysis (with no postenrollment exclusions), although it is rare that even prospective studies capture deaths before hospital arrival. In a trial of patients with TBI randomly assigned to undergo immediate versus delayed craniectomy, the importance of including patients unable to survive long enough to undergo their assigned delayed craniectomy would be clear. Naturally, there is sufficient physiological rationale for avoiding delayed craniectomy to make such a trial unethical. Observational studies such as ours are the only method of addressing the question, but they must make every effort to avoid falling into this immortal time-bias trap.

Within the US military trauma system, comprehensive data (including the dates and times of key events) were captured for a large, representative sample of patients who underwent craniectomy. This enabled a statistically powerful as well as valid survival analysis. As in previous observational studies, patients were selected on the basis of having undergone neurosurgery. In contrast to the previous research, however, this study minimized immortal time bias by 1) entering patients into the Cox survival analyses upon their arrival in the ED of the hospital in which craniectomy was performed (time 0 ); 2) defining each patient's start of craniectomy as a time-dependent covariate; and 3) including prehospital delays among the key covariates for adjustment.

Another unique aspect of this study was the evaluation of the elapsed time from injury to neurosurgery in quintiles to produce more interpretable and clinically useful results. Delays from injury to neurosurgery were inversely correlated with head injury severity, due almost entirely to the in-hospital, not prehospital, delays. On the other hand, the increased mortality risk for delays greater than 5.33 hours was due mostly to prehospital rather than in-hospital delays. These findings add evidence to help resolve the prevailing debate and guide the development and al- location of critical but limited transport and neurosurgical resources within a trauma system.

Arrival at a facility with neurosurgical capability ensures the availability of neurocritical care and optimal medical treatment in addition to surgery when indicated. Patients can be thoroughly assessed at baseline and monitored with CT scans, ICP measurements, and serial neurological examinations to precisely diagnose indications for neurosurgical intervention, whether they are immediately detectable or evolving slowly over time. Findings from this study suggest a greater and more urgent need to reduce a patient's prehospital rather than in-hospital delay to neurosurgery. In addition, continued efforts to improve neurological care in the prehospital environment could potentially mitigate the effect of unavoidable delays to neurosurgical intervention.

\section{Limitations of the Study}

The exact indications for craniectomy were not always apparent and included a heterogeneous mix of TBI with space-occupying hematomas, diffuse cerebral edema, and displaced skull fractures from both blunt and penetrating mechanisms. It is possible that specific diagnoses may be more responsive to medical or surgical management, and further study is needed to determine the optimal treatment for each. However, within the military trauma system where CT scanning is usually not available until arrival at a role 3 facility, early decision-making often must occur without the benefit of imaging studies.

Other sources of residual or unmeasured but potentially important confounding were possible. The study had no access to autopsy data, and injury severity may have been underestimated, particularly for early decedents who may not have survived long enough for definitive injury diagnosis in all body regions. For example, the maximum head AIS score and ISS assigned to the 6 earliest decedents (within $<8$ hours of hospital admission) did not differ, on average, from the respective values for the 12 longest-surviving decedents with $>5$ in-hospital days $(\mathrm{p}=0.51, \mathrm{p}=$ 0.71 , respectively).

Longer-term survival (e.g., 30 days) could not be assessed in the current study because only in-hospital survival times were known, and non-US military survivors were often transferred to local national hospitals, precluding further follow-up. Time to craniectomy is likely to be a determinant of morbidity as well as mortality, but information on functional outcomes among survivors was not available in this study.

\section{Conclusions}

We described the impact of delay to craniectomy on survival in a retrospective study of postcraniectomy patients with severe combat-related TBI. Postoperative mortality was significantly lower when craniectomy was initiated within 5.33 hours of injury relative to longer delays. Prehospital rather than in-hospital delays to surgery were most closely associated with postcraniectomy mortality. In addition to expediting prehospital transport to neurosurgery, additional efforts to optimize medical management and physiological stabilization during point of injury care, 
en route care, and initial surgical stabilization of severe TBI may help mitigate the effect of longer delays to definitive care. Future research seeking to optimize craniectomy timing is needed and should ascertain all key time points (injury, diagnosis of the indication for surgery, hospital arrival, start of surgery, and death or hospital discharge) as well as all patients eligible for the procedure, including those who die before craniectomy can be initiated. The inclusion of craniectomy-eligible patients whose death precludes the procedure will be especially critical in future studies seeking to evaluate whether earlier craniectomy by non-neurosurgeons might be preferable to longer delays to reach a neurosurgeon. Ideally, information on functional outcome in addition to mortality should guide recommendations.

\section{Acknowledgments}

This research was supported in part by Dr. del Junco's appointment to the Faculty Research Participation Program at the US Army Institute of Surgical Research administered by the Oak Ridge Institute for Science and Education through an interagency agreement between the US Department of Energy and the US Army Medical Research and Materiel Command (USAMRMC).

\section{References}

1. Barthélemy EJ, Melis M, Gordon E, Ullman JS, Germano IM: Decompressive craniectomy for severe traumatic brain injury: a systematic review. World Neurosurg 88:411-420, 2016

2. Bell RS, McCafferty R, Shackelford S, Tomlin J, Dirks M, Neal C, et al: Emergency life-saving cranial procedures by non-neurosurgeons in deployed settings (CPG ID:68), in Joint Trauma System Clinical Practice Guidelines. Arlington, VA: Department of Defense Center of Excellence for Trauma, 2018 (http://jts.amedd.army.mil/assets/docs/cpgs/ JTS_Clinical_Practice_Guidelines_(CPGs)/Emergency_ Cranial_Procedures_by_Non-Neurosurgeons_Deployed Setting_23_Apr_2018_ID68.pdf) [Accessed October 3, 2018]

3. Bricolo AP, Pasut LM: Extradural hematoma: toward zero mortality. A prospective study. Neurosurgery 14:8-12, 1984

4. Cooper DJ, Rosenfeld JV, Murray L, Arabi YM, Davies AR, D'Urso P, et al: Decompressive craniectomy in diffuse traumatic brain injury. N Engl J Med 364:1493-1502, 2011

5. Copes WS, Staz CF, Konvolinka CW, Sacco WJ: American College of Surgeons audit filters: associations with patient outcome and resource utilization. J Trauma 38:432-438, 1995

6. De Vloo P, Nijs S, Verelst S, van Loon J, Depreitere B: Prehospital and intrahospital temporal intervals in patients requiring emergent trauma craniotomy. A 6-year observational study in a level 1 trauma center. World Neurosurg 114:e546-e 558, 2018

7. Dent DL, Croce MA, Menke PG, Young BH, Hinson MS, Kudsk KA, et al: Prognostic factors after acute subdural hematoma. J Trauma 39:36-43, 1995

8. DuBose JJ, Barmparas G, Inaba K, Stein DM, Scalea T, Cancio LC, et al: Isolated severe traumatic brain injuries sustained during combat operations: demographics, mortality outcomes, and lessons to be learned from contrasts to civilian counterparts. J Trauma 70:11-18, 2011

9. Eastridge BJ, Hardin M, Cantrell J, Oetjen-Gerdes L, Zubko T, Mallak C, et al: Died of wounds on the battlefield: causation and implications for improving combat casualty care. J Trauma 71 (1 Suppl):S4-S8, 2011

10. Eastridge BJ, Mabry RL, Seguin P, Cantrell J, Tops T, Uribe P, et al: Death on the battlefield (2001-2011): implications for the future of combat casualty care. J Trauma Acute Care Surg 73 (6 Suppl 5):S431-S437, 2012

11. Fountain DM, Kolias AG, Lecky FE, Bouamra O, Lawrence T, Adams H, et al: Survival trends after surgery for acute subdural hematoma in adults over a 20 -year period. Ann Surg 265:590-596, 2017

12. Gutterman P, Shenkin HA: Prognostic features in recovery from traumatic decerebration. J Neurosurg 32:330-335, 1970

13. Haselsberger K, Pucher R, Auer LM: Prognosis after acute subdural or epidural haemorrhage. Acta Neurochir (Wien) 90:111-116, 1988

14. Hatashita S, Koga N, Hosaka Y, Takagi S: Acute subdural hematoma: severity of injury, surgical intervention, and mortality. Neurol Med Chir (Tokyo) 33:13-18, 1993

15. Howard MA III, Gross AS, Dacey RG Jr, Winn HR: Acute subdural hematomas: an age-dependent clinical entity. J Neurosurg 71:858-863, 1989

16. Hutchinson PJ, Kolias AG, Timofeev IS, Corteen EA, Czosnyka M, Timothy J, et al: Trial of decompressive craniectomy for traumatic intracranial hypertension. N Engl J Med 375:1119-1130, 2016

17. Jamieson KG, Yelland JD: Extradural hematoma. Report of 167 cases. J Neurosurg 29:13-23, 1968

18. Kim YJ: The impact of time to surgery on outcomes in patients with traumatic brain injury: a literature review. Int Emerg Nurs 22:214-219, 2014

19. Koç RK, Akdemir H, Oktem IS, Meral M, Menkü A: Acute subdural hematoma: outcome and outcome prediction. Neurosurg Rev 20:239-244, 1997

20. Kolias AG, Adams H, Timofeev I, Czosnyka M, Corteen EA, Pickard JD, et al: Decompressive craniectomy following traumatic brain injury: developing the evidence base. Br J Neurosurg 30:246-250, 2016

21. Kotwica Z, Brzeziński J: Acute subdural haematoma in adults: an analysis of outcome in comatose patients. Acta Neurochir (Wien) 121:95-99, 1993

22. Kvarnes TL, Trumpy JH: Extradural haematoma. Report of 132 cases. Acta Neurochir (Wien) 41:223-231, 1978

23. Lee EJ, Hung YC, Wang LC, Chung KC, Chen HH: Factors influencing the functional outcome of patients with acute epidural hematomas: analysis of 200 patients undergoing surgery. J Trauma 45:946-952, 1998

24. Marshall SA, Bell R, Armonda RA, Savitsky E, Ling GSF: Management of moderate and severe TBI, in Tsao JW (ed): Traumatic Brain Injury. New York: Springer, 2012, pp 69-87

25. Massaro F, Lanotte M, Faccani G, Triolo C: One hundred and twenty-seven cases of acute subdural haematoma operated on. Correlation between CT scan findings and outcome. Acta Neurochir (Wien) 138:185-191, 1996

26. Matsushima K, Inaba K, Siboni S, Skiada D, Strumwasser AM, Magee GA, et al: Emergent operation for isolated severe traumatic brain injury: does time matter? J Trauma Acute Care Surg 79:838-842, 2015

27. McCafferty R, Neal C, Marshall S, Pamplin J, Bell R, Rivet D, et al: Neurosurgery and severe head injury (CPG ID:30), in Joint Trauma System Clinical Practice Guidelines. Arlington, VA: Department of Defense Center of Excellence for Trauma, 2017 (http://jts.amedd.army.mil/assets/docs/cpgs/ JTS_Clinical_Practice_Guidelines_(CPGs)/Neurosurgery_ Severe_Head_Injury_02_Mar_2017_ID30.pdf) [Accessed October 3, 2018]

28. Mendelow AD, Karmi MZ, Paul KS, Fuller GA, Gillingham FJ: Extradural haematoma: effect of delayed treatment. BMJ 1:1240-1242, 1979

29. Phuenpathom N, Choomuang M, Ratanalert S: Outcome and outcome prediction in acute subdural hematoma. Surg Neurol 40:22-25, 1993 
30. Poon WS, Li AK: Comparison of management outcome of primary and secondary referred patients with traumatic extradural haematoma in a neurosurgical unit. Injury 22:323325,1991

31. Royal College of Surgery of England: Report of the Working Party on the Management of Patients With Head Injuries. London: RCS, 1999

32. Seelig JM, Becker DP, Miller JD, Greenberg RP, Ward JD, Choi SC: Traumatic acute subdural hematoma: major mortality reduction in comatose patients treated within four hours. N Engl J Med 304:1511-1518, 1981

33. Sergides IG, Whiting G, Howarth S, Hutchinson PJ: Is the recommended target of 4 hours from head injury to emergency craniotomy achievable? Br J Neurosurg 20:301-305, 2006

34. Spaite DW, Hu C, Bobrow BJ, Chikani V, Barnhart B, Gaither JB, et al: Association of out-of-hospital hypotension depth and duration with traumatic brain injury mortality. Ann Emerg Med 70:522-530, 530.e1, 2017

35. Spaite DW, Hu C, Bobrow BJ, Chikani V, Barnhart B, Gaither JB, et al: The effect of combined out-of-hospital hypotension and hypoxia on mortality in major traumatic brain injury. Ann Emerg Med 69:62-72, 2017

36. Spaite DW, Hu C, Bobrow BJ, Chikani V, Sherrill D, Barnhart B, et al: Mortality and prehospital blood pressure in patients with major traumatic brain injury: implications for the hypotension threshold. JAMA Surg 152:360-368, 2017

37. Stone JL, Lowe RJ, Jonasson O, Baker RJ, Barrett J, Oldershaw JB, et al: Acute subdural hematoma: direct admission to a trauma center yields improved results. J Trauma 26:445450, 1986

38. Stone JL, Rifai MH, Sugar O, Lang RG, Oldershaw JB, Moody RA: Subdural hematomas. I. Acute subdural hematoma: progress in definition, clinical pathology, and therapy. Surg Neurol 19:216-231, 1983

39. Taussky P, Widmer HR, Takala J, Fandino J: Outcome after acute traumatic subdural and epidural haematoma in Switzerland: a single-centre experience. Swiss Med Wkly 138:281285,2008

40. Tian HL, Chen SW, Xu T, Hu J, Rong BY, Wang G, et al: Risk factors related to hospital mortality in patients with isolated traumatic acute subdural haematoma: analysis of 308 patients undergone surgery. Chin Med J (Engl) 121:1080 1084,2008

41. Tien HC, Jung V, Pinto R, Mainprize T, Scales DC, Rizoli SB: Reducing time-to-treatment decreases mortality of trauma patients with acute subdural hematoma. Ann Surg 253:1178-1183, 2011

42. Walcott BP, Khanna A, Kwon CS, Phillips HW, Nahed BV, Coumans JV: Time interval to surgery and outcomes following the surgical treatment of acute traumatic subdural hematoma. J Clin Neurosci 21:2107-2111, 2014

43. Wilberger JE Jr, Harris M, Diamond DL: Acute subdural hematoma: morbidity and mortality related to timing of operative intervention. J Trauma 30:733-736, 1990

44. Wilberger JE Jr, Harris M, Diamond DL: Acute subdural hematoma: morbidity, mortality, and operative timing. J Neurosurg 74:212-218, 1991

45. Zafrullah Arifin M, Gunawan W: Analysis of presurgery time as a prognostic factor in traumatic acute subdural hematoma. J Neurosurg Sci 57:277-280, 2013

\section{Disclosures}

The authors report no conflict of interest concerning the materials or methods used in this study or the findings specified in this paper.

\section{Author Contributions}

Conception and design: Shackelford, del Junco, Reade, Bell. Acquisition of data: Shackelford, del Junco. Analysis and interpretation of data: Shackelford, del Junco, Reade. Drafting the article: Shackelford, del Junco. Critically revising the article: all authors. Reviewed submitted version of manuscript: all authors. Approved the final version of the manuscript on behalf of all authors: Shackelford. Statistical analysis: del Junco.

\section{Supplemental Information \\ Videos \\ Video Abstract. https://vimeo.com/298582139.}

Previous Presentations

Portions of this work were used in an oral presentation at the Military Health Services Research Symposium, held in Kissimmee, FL, on August 20-23, 2018.

\section{Correspondence}

Stacy A. Shackelford: DHA, Combat Support, Ft. Sam Houston, San Antonio, TX. stacy.a.shackelford.mil@mail.mil. 